\title{
Influence of the network structure on robustness
}

\author{
A. Jamakovic and S. Uhlig \\ Faculty of Electrical Engineering, Mathematics, and Computer Science, \\ Delft University of Technology, The Netherlands
}

\begin{abstract}
The classical connectivity is typically used to capture the robustness of networks. Robustness, however, encompasses more than this simple definition of being connected. A spectral metric, referred to as the algebraic connectivity, plays a special role for the robustness since it measures the extent to which it is difficult to cut the network into independent components. We rely on the algebraic connectivity to study the robustness to random node and link failures in three important network models: the random graph of Erdôs-Rènyi, the small-world graph of Watts and Strogatz and the scale-free graph of BarabásiAlbert. We show that the robustness to random node and link failures significantly differs between the three models. This points to explicit influence of the network structure on the robustness. The homogeneous structure of the random graph of Erdôs-Rènyi implies an invariant robustness under random node failures. The heterogeneous structure of the small-world graph of Watts and Strogatz and scale-free graph of Barabási-Albert, on the other hand, implies a non-trivial robustness to random node and link failures.
\end{abstract}

\section{INTRODUCTION}

Robustness of the network topology is a key aspect in the design of computer networks. Network design heuristics [6] that are typical for computer networks, rely for a large part on the classical connectivity [13]. Network robustness, however, encompasses more than just providing an answer to questions of the form "What is the maximum number of node or link failures a network can accommodate before it becomes disconnected?" [12]. Here, we rely on a spectral metric, referred to as the algebraic connectivity [8], that has been proven to be a distinguishable parameter in many robustness related problems [16]. By considering the algebraic connectivity as a measure of the network robustness, we show that the structure, not only the classical connectivity, has a clear influence on the robustness of the network topology.

In this paper we show that for a thorough understanding of robustness, a proper knowledge of the topological structure of certain classes of networks is extremely important. Furthermore, we show that the type of random failure is highly predictable since the robustness to random node and link failures differs significantly between certain classes of networks. For that reason, we take network classes that have two structurally opposite underlying mechanisms: the homogeneous structure of the random graph of Erdős-Renyi versus the heterogeneous structure of the scale-free graph of BarabásiAlbert. In addition, we consider the small-world graph of Watts and Strogatz as its exhibits the properties of both the random graph and the scale-free model. These three models are widely used for modeling the topology of computer networks [1] [17].

This research was supported by the Next Generation Infrastructures programme (www.nginfra.nl)
The homogeneous structure of the random graph of ErdôsRenyi implies an invariant robustness under random node and link failures. The heterogeneous structure of the scale-free graph of Barabási-Albert, on the other hand, implies a non trivial robustness to random node and link failures. Such a theoretically explained behavior was also confirmed for the small-world graph of Watts-Strogatz.

The remainder of this paper is structured as follows. Section II presents the theoretical background on the algebraic connectivity. Section III gives an analytical result linking the classical connectivity to the algebraic connectivity. Section IV provides simulation results on the distribution of the algebraic connectivity in the three network models subject to random failures. Finally, Section V concludes and discusses further work.

\section{BACKGROUND}

A network is represented as an undirected graph $G=$ $(\mathcal{N}, \mathcal{L})$ where $\mathcal{N}$ denotes the set of nodes and $\mathcal{L}$ the set of links, with $N=|\mathcal{N}|$ nodes and $L=|\mathcal{L}|$ links, respectively [4]. Before giving an overview of theoretical results on the algebraic connectivity, we define the Laplacian matrix associated with a graph.

The Laplacian matrix of $G$ with $N$ nodes is an $N \times N$ matrix $Q=\Delta-A$ where $\Delta=\operatorname{diag}\left(d_{i}\right), d_{i}$ is the nodal degree of node $i \in \mathcal{N}$ and $A$ is the adjacency matrix of $G$ [14] The eigenvalues of the Laplacian matrix $\lambda_{1} \geq \ldots \geq \lambda_{N-1} \geq \lambda_{N}=$ 0 are all real and nonnegative [9][15]. The second smallest eigenvalue of the standard Laplacian matrix, as proposed by Fiedler [8], is called the algebraic connectivity. The following results can be found [10] on the algebraic connectivity $\lambda_{N-1}$ :

1) $0 \leq \lambda_{N-1} \leq \frac{N}{N-1} \min \left\{d_{i}: i \in \mathcal{N}\right\}$ : if a graph $G$ is a complete graph $K_{N}$, then $\lambda_{N-1}=N$ and if $G$ is a disconnected graph, then $\lambda_{N-1}=0$.

2) If $\lambda_{N-i+1}=0$ and $\lambda_{N-1} \neq 0$, then $G$ has exactly $i$ components.

3) If $G_{1}=\left(V, L_{1}\right)$ is a subgraph of $G$, then $\lambda_{N-1}\left(G_{1}\right) \leq$ $\lambda_{N-1}(G)$.

Point 1) says that the algebraic connectivity is upper bounded by $\frac{N}{N-1}$ times the minimal nodal degree of the graph. Point 2) says that the multiplicity of zero as an eigenvalue corresponds to the number of components of the graph. Point 3) says that if a subgraph $G_{1}$ of a graph $G$ has all the nodes of $G$ and a subset of the links of $G$, then the algebraic connectivity of $G_{1}$ is smaller than the one of $G$.

Let us also introduce two connectivity metrics of $G: 1$ ) for $k \geq 1$, a graph $G$ is (node) $k$-connected if either $G$ is a complete graph $K_{k+1}$ or it has at least $k+2$ nodes and no set 
of $k-1$ nodes that separates it, 2) for $k \geq 1$ a graph $G$ is $k$ link connected if it has at least two nodes and no set of at most $k-1$ links that separates it. The maximum value of $k$ for which a connected graph is $k$-connected equals the node connectivity $\kappa_{N}$. The link connectivity $\kappa_{L}$ is defined analogously [4]. The node (link) connectivity of an incomplete graph $G \neq K_{N}$ is at least as large as the algebraic connectivity $\lambda_{N-1} \leq \kappa_{N} \leq \kappa_{L}$ [8]. If $G=K_{N}$, then $\lambda_{N-1}=N>\kappa_{N}=N-1$.

\section{LINKING $k$-CONNECTIVITY AND THE ALGEBRAIC CONNECTIVITY}

In this section we present an analytic result that allows us to determine the values of network model parameters that lead to identical algebraic connectivity distributions. We start by discussing the considered network model and a set of classical theorems corresponding to it. We analyze then the distributions of the algebraic connectivity on this network model.

\section{A. Random graph of Erdös and Rènyi}

The random graph, by Erdôs and Rènyi, is the simplest model to describe the topology of a network. We denote the random graph by $G_{p}(N)$, where $N$ is the number of nodes in the graph and $p$ is the probability of having a link between any two nodes (or shortly the link probability). $G_{p}(N)$ is the set of all such graphs in which a graph having $L$ links appears with probability $p^{L}(1-p)^{L_{\max }-L}$, where $L_{\max }$ is the maximum possible number of links. Many properties of the random graph are known analytically in the limit of large graph size $N$, as was shown by Erdôs and Rènyi in a series of papers in the 1960s and later by Bollobás [5]. Typically, for large graph size $N$, the degree distribution of the random graph model, which is a binomial distribution, can be replaced by a Poisson distribution, i.e.

$$
\operatorname{Pr}[D=k]=\left(\begin{array}{c}
N-1 \\
k
\end{array}\right) p^{k}(1-p)^{N-1-k} \simeq \frac{\mu^{k} e^{-\mu}}{k !}
$$

where $\mu=p(N-1)$ equals the mean nodal degree $E[D]$.

The expected structure of the random graph varies with the value of $p$. For our purposes the most important property of the random graph is that it possesses a phase transition [7]: from a low-density, low $p$ value for which there are few links and many small components, having an exponential size distribution and finite mean size, to a high-density, high $p$ value for which an extensive fraction of all nodes are joined together in a single giant component, the remainder of the nodes occupying smaller components with again an exponential size distribution and finite mean size. This phase transition is studied in detail by Janson et al. in [11] and given with the following connectivity theorem.

Theorem 1. If we start with a random graph on $N$ nodes and an empty link set and add links randomly and independently one by one until having $L$ links, the graph almost surely becomes 1 -connected when $L \geq \frac{N \log N}{2}+O(N)$. Since $p=\frac{L}{\left(\begin{array}{c}N \\ 2\end{array}\right)}$ we can say that for a random graph to be 1 -connected the following must hold, for large $N$,

$$
p \geq \frac{\log N}{N} \equiv p_{c}
$$

From the above theorem, the fundamental result [7] can be deduced that the probability of a random graph being connected is about the probability that it has no node of degree zero. Given the importance of this result we also give the following theorem, which relates the $k$-connectivity to the minimum nodal degree [5].

Theorem 2. If links are added one by one to the empty random graph of $N$ nodes in an order chosen uniformly and at random from the $\left(\begin{array}{c}N \\ 2\end{array}\right)$ ! possibilities, then almost surely the resulting graph becomes $k$-connected when it achieves a minimum degree of $k$. In other words, for large $N$,

$$
\operatorname{Pr}\left[G_{p}(N) \text { is } k \text {-connected }\right]=\operatorname{Pr}\left[d_{\min } \geq k\right]
$$

where $d_{\min }$ is the minimum nodal degree.

\section{B. Distribution of the algebraic connectivity}

To understand the probability distribution of the algebraic connectivity $\lambda_{N-1}$, we will use the second theorem of connectivity to deduce the probability $p$ of the presence of a link between any two nodes in the Erdós and Renyi random graph. From (3) and (1), we have that the probability of $k$ connectivity in $G_{p}(N)$ equals

$$
\operatorname{Pr}\left[G_{p}(N) \text { is } k \text {-connected }\right]=\left(1-\sum_{m=0}^{k-1} \frac{(\mu)^{m} e^{-\mu}}{m !}\right)^{N} \text {. }
$$

Solving the above equation for a given probability of being $k$-connected and a given number of nodes $N$, one can easily find the probability $p$ of the presence of a link between any two nodes in $G_{p}(N)$. In this paper, we use two arbitrary probabilities, i.e. 0.5 and 0.9 , of a graph being $k$-connected to deduce $p$. The deduced probabilities of being $k$-connected are listed in Table I. Figure 1 relates, for different values of $N$, the probabilities of 1-connectivity and 10-connectivity to the link probability $p$. We observe on Figure 1 that larger graph sizes require smaller link probabilities $p$ to have a given probability of being $k$-connected.

Figure 2 includes the simulation results on $G_{p}(N)$ with $N=50$ and 400 and the corresponding link probabilities $p$ (equation 4). From this Figure, we can observe that, for a given probability of the graph's $k$-connectivity, the algebraic connectivity $\lambda_{N-1}$ has the same probability distribution for different graph sizes $N$. Figure 2 suggests that the probability of being $k$-connected might define robustness classes, as they seem to correspond to a particular density of the algebraic connectivity. Although it is proven to be a distinguishable parameter in many robustness related problems [16], today not much is known about the practical meaning of the algebraic connectivity. It was shown in [12] that there is no trivial connection between the algebraic connectivity and the connectivity to node and link failures, i.e. the node $\kappa_{N}$ and link $\kappa_{L}$ connectivity. Furthermore, [12] showed that the relationship between the algebraic connectivity and the connectivity to node and link failures depends on the considered network model. Here we see that, at least in distributional terms, the algebraic connectivity seems to provide a signature of the probability of being $k$-connected. This result points towards the possibility of defining robustness classes based on the algebraic connectivity. 
TABLE I

THE LINK PROBABILITY $p$ IN $G_{p}(N)$ WITH $N=50,100,200$ AND 400, FOR VALUES OF THE PROBABILITY OF BEING 1 -CONNECTED AND 10-CONNECTED OF 0.5 AND 0.9 .

\begin{tabular}{|l|l|l|l|l|}
\hline $\operatorname{Pr}[k$-connectivity $]$ & $N=50$ & $N=100$ & $N=200$ & $N=400$ \\
\hline $\operatorname{Pr}[1$-connectivity $]=0.5$ & $p=0.0875$ & $p=0.0503$ & $p=0.0285$ & $p=0.0159$ \\
\hline $\operatorname{Pr}[1$-connectivity $]=0.9$ & $p=0.1258$ & $p=0.0693$ & $p=0.0379$ & $p=0.0207$ \\
\hline $\operatorname{Pr}[10$-connectivity $]=0.5$ & $p=0.3715$ & $p=0.1963$ & $p=0.1036$ & $p=0.0546$ \\
\hline $\operatorname{Pr}[10$-connectivity $]=0.9$ & $p=0.4378$ & $p=0.2280$ & $p=0.1189$ & $p=0.0620$ \\
\hline
\end{tabular}

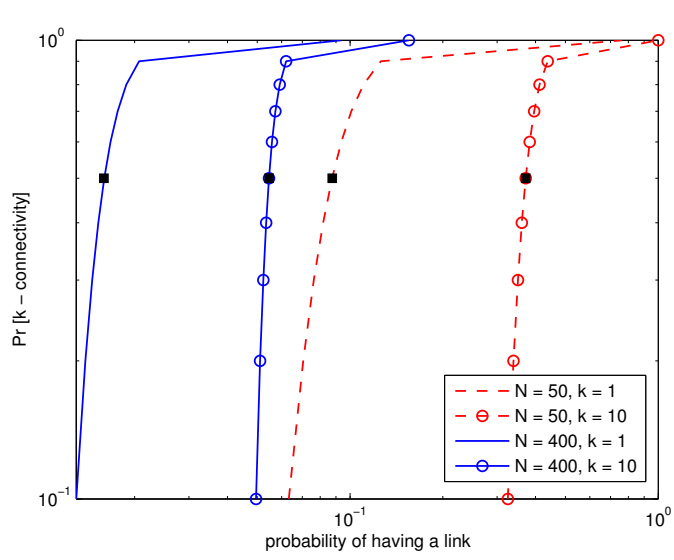

Fig. 1. The probability of being 1-connected and 10-connected as a function of the link probability $p$ in $G_{p}(N)$ with $N=50$ and 400 . Black markers indicate the values of the link probability $p$ for wich the probability of being 1 -connected and 10-connected equals 0.5 . These values are also presented in Table I.
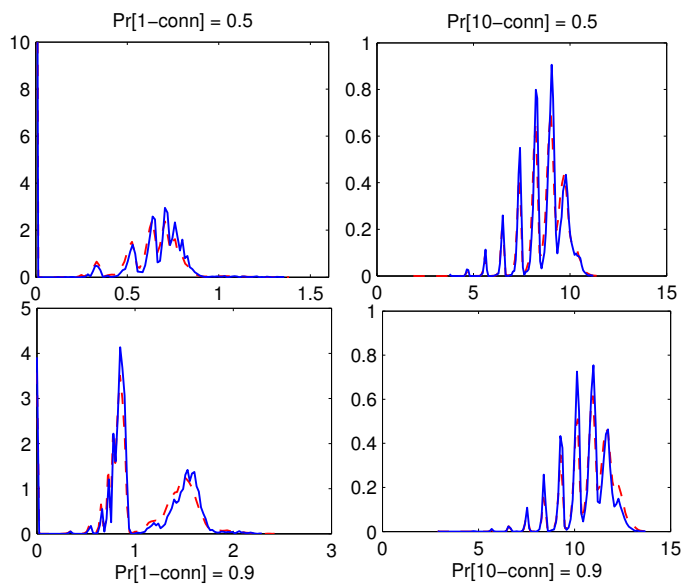

Fig. 2. The probability density function of the algebraic connectivity $\lambda_{N-1}$ in $G_{p}(N)$ with $N=50$ (dotted red line), 400 (full blue line) and the link probability $p$, which is deduced from the probability of $G_{p}(N)$ beeing $k$ connected.

\section{BEHAVIOR OF THE ALGEBRAIC CONNECTIVITY UNDER RANDOM FAILURES}

In this section we present simulation results on the distribution of the algebraic connectivity. We first discuss the considered network models. Then we analyze simulation results where we apply topological changes in the form of random node removal.

\section{A. Random Graph of Erdös-Rényi}

In the previous section we have seen that in the Erdôs-Rényi random graph, the parameters of interest are $N$ and $p$. We simulate for each combination of $N$ and $p, 10^{3}$ independent $G_{p}(N)$ graphs. The number of nodes is 250 and the link probability takes one of the following two values, $p=0.008$ and $p=0.016$. From each combination of $N$ and $p$, we compute the algebraic connectivity $\lambda_{N-1}$ and sort the $10^{3}$ values in increasing order. We plot them so that the $i^{\text {th }}$ smallest algebraic connectivity value $\lambda_{N-1}^{i}, 1 \leq i \leq 10^{3}$, is drawn at $(x, y)$ with $x=\frac{(i-1)}{\left(10^{3}-1\right)}$ and $y=\lambda_{N-1}^{i}$. In this way all values in the $\mathrm{x}$-axis are in the $[0,1]$ range. In Figure 3 , the distribution of the algebraic connectivity is given for the Erdôs-Rényi random graph with $N=250$ and $p=0.008$. Note that considered values of the link probability $p$ are smaller than the value for which a random graph with $N=250$ almost surely becomes connected (equation 2). For that reason, we compute the algebraic connectivity of the largest connected component. We also compute the distribution when the random graph is affected by random node failures: in two consecutive steps, we randomly remove a node and all its connections and compute the algebraic connectivity from the remaining largest connected component. Figure 3 shows that the random graph subject to random node removal, exhibits a slight decrease in the distribution of the algebraic connectivity. A similar behavior has been observed for the random graph with twice the number of links (not shown due to space limitations). The analytical results presented in Section II explain the decrease in the algebraic connectivity under the condition that removal of links would negatively effect the link density of the resulting graph (third result on the algebraic connectivity). Taking this result into account, it seems that the random removal of nodes leaves a subgraph that has approximately the same (or a slightly smaller) proportion of nodes to links as the original graph. Figure 4 supports this observation by showing that nodes to links ratios after one or two node removals are the same. Consequently, the removal of random nodes results in a subgraph that has approximately the same structure as the random graph upon which the changes were applied.

\section{B. Small-World Graph of Watts-Strogatz}

The small-world model describes the fact that despite the large graph size, in most real-world networks there is a relatively short path between any two nodes. There are different realizations of the small-world model, but the original model as proposed by Watts and Strogatz [19] is by far the most widely studied. It starts by building the ring $R_{N}$ with $N$ nodes, and then joining each node to $2 s$ neighbors ( $s$ on either side of the ring). This results in the ring lattice $C(N, s)$ with $L=s N$ links. The small-world graph is then created by moving, with probability $p_{r}$, one end of each link (connected to a clockwise neighbor) to a new node chosen uniformly in the ring lattice, except that no double links or loops are allowed. The rewiring 


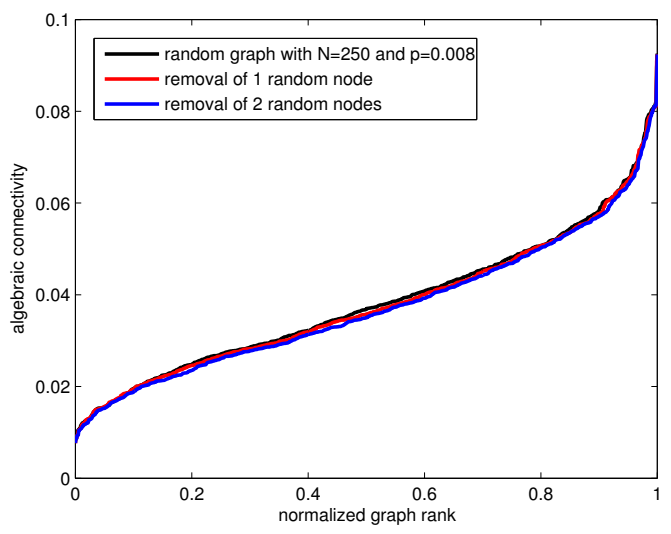

Fig. 3. Distribution of the algebraic connectivity for the random graph of Erdôs-Rényi with 250 nodes and a link probability $p=0.008$. The distribution of the algebraic connectivity is also measured when the model is subject to topological changes in the form of random node removal.
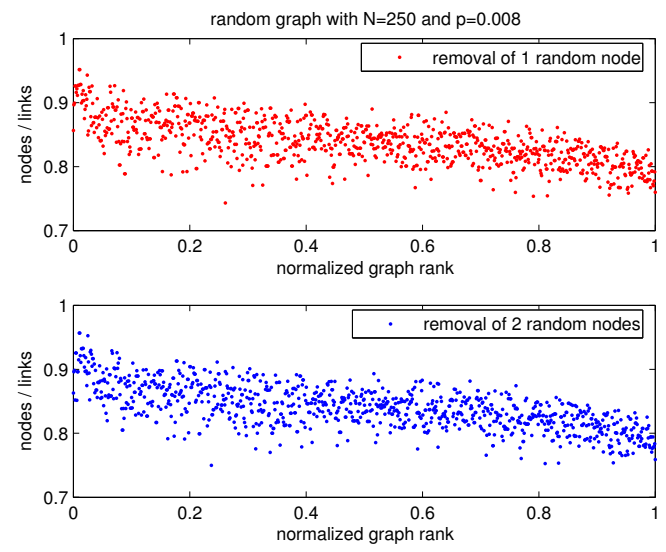

Fig. 4. The ratio of the number of nodes to the number of links after the random graph of Erdôs-Rényi with 250 nodes and $p=0.008$ is subject to the topological changes in the form of random node removal.

process allows the small-world model to interpolate between a regular lattice $\left(p_{r}=0\right)$ and something which is similar, though not identical, to a random graph $\left(p_{r}=1\right)$. For already small $p_{r}$ the small-world becomes a locally clustered network in which two arbitrary nodes are connected by a small number of intermediate links [19].

We simulate, for $N$ and $s, 10^{3}$ independent Watts-Strogatz small-world graphs. The number of nodes $N$ is 250 and $s$ takes one of the following two values, $s=1$ and $s=2$. The rewiring probability is set to be $p_{r}=0.008$. Similarly to results for the Erdôs-Rényi random graph, we plot in Figure 5 the distribution of the algebraic connectivity as a function of the normalized graph rank. We also compute and plot the distribution when the small-world network is affected by random node failures: in two consecutive steps, we randomly remove a node and all its connections and compute the algebraic connectivity from the remaining largest connected component.

From Figure 5 we observe that the value of the algebraic connectivity is constant for nearly all simulated small-world graphs. We also observe that the higher value of the algebraic connectivity is a consequence of a graph with a higher value of the link density: for $N=250$ and $s=2$, the small-world

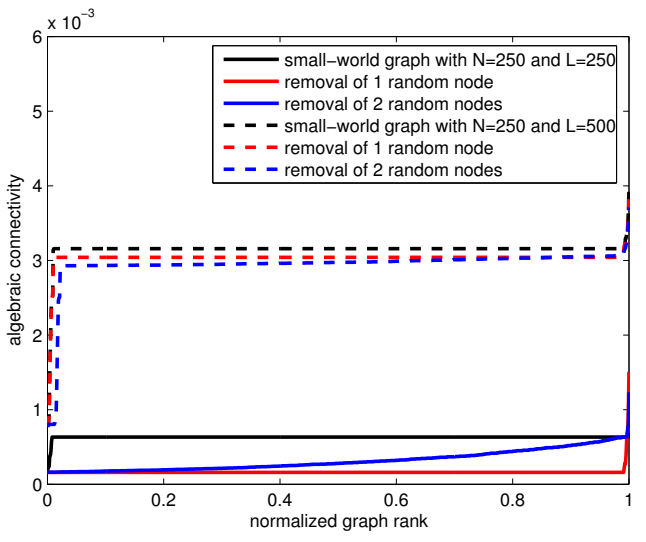

Fig. 5. Distribution of the algebraic connectivity for the small-world of WattsStrogatz with 250 nodes and 250/500 links. The distribution of the algebraic connectivity is also measured when the model is subject to topological changes in the form of random node removal.

graph has twice as much links, i.e. $L=500$, as the smallworld with $N=250$ and $s=1$. The constant behavior of the algebraic connectivity comes from the fact that the small-world process introduces $p_{r} N s$ non-lattice links, which for $p_{r}=0.008$ results in only few rewired links. Therefore, resulting small-world graphs are almost regular ring lattices so that their values of the algebraic connectivity are constant for almost all simulated graphs. Furthermore, the removal of random nodes in a nonsparse small-wold graph (see dashed lines in Figure 5) results in a slight decrease in the distribution of the algebraic connectivity. Then, the remaining largest connected component have the same proportion of nodes to links, i.e. $\frac{N}{L}=0.5$, as the initial small-world graph (not shown due to space limitation).

On the other hand, for the sparse small-world graph, i.e. $L=N$, the distribution of the algebraic connectivity is surprisingly different. After random removal of one node, there seems to be a substantial decrease in the robustness of the remaining largest connected component. However, after random removal of two nodes, the distribution of the algebraic connectivity is likely to be increasing. Take notice of an unchanged ratio between the number of nodes and the number of links, i.e. the ratio after random removal of one or two nodes is equal to the ratio in a given small-world graph with $N=L$. As evident from the figure, this does not mean that remaining largest connected components after random removal of nodes will have the same robustness as the original graph. While this may appear inconsistent with prior results, it can be easily understood: random removal of nodes in a practically regular graph, i.e. here the ring graph with small number of rewired links, most likely fragments the small-world so that in the remaining largest connected component a highly regular structure is observed again. This process is repeated until the network is fragmented in such a way that the regularity is hardly perceptible.

\section{Scale-Free Graph of Barabási-Albert}

Scale-free models have a power-law degree distribution which contrasts with that of random or small-world graphs. Barabási [2] showed that growth and preferential attachment 


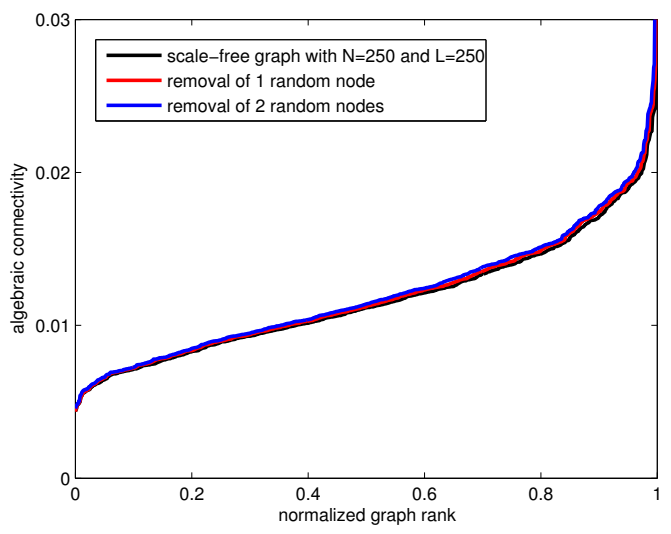

Fig. 6. Distribution of the algebraic connectivity for the scale-free graph of Barabási-Albert with 250 nodes and 250 links, with and without random nodes removal.

of nodes, which implies that the nodes with larger degree are more likely candidates for attachment of new nodes, give rise to a class of graphs with a power-law degree distribution. The Barabási-Albert model starts with a small number $m_{0}$ of fullymeshed nodes, followed at every step by a new node attached to $m \leq m_{0}=2 m+1$ nodes already present in the system. After $t$ steps this procedure results in a graph with $N=t+m_{0}$ nodes and $L=\frac{m_{0}\left(m_{0}-1\right)}{2}+m t$ links.

We simulate, for $N$ and $m, 10^{3}$ independent BarabásiAlbert scale-free graphs. The number of nodes is 250 and the parameter $m$ takes one of the following two values, $m=1$ and $m=2$. For each combination of $N$ and $m$, we compute the algebraic connectivity $\lambda_{N-1}$, and sort the $10^{3}$ independent values in increasing order. We plot them in the same way as in the simulations for the Erdôs-Rényi and Watts-Strogatz graph. In Figures 6 and 8, the distribution of the algebraic connectivity is given, respectively for the Barabási-Albert scale-free graph with $m=1$ and with $m=2$. Note that for the Barabási-Albert scale-free with $m=2$, the number of links, i.e. $L=500$, is twice as large as for the BarabásiAlbert scale-free with $m=1$, i.e. $L=250$. Along with the standard behavior of the algebraic connectivity, we also show the distribution when the model is exposed to the random node removal: in two consecutive steps, we randomly remove a node and all its connections and calculate the algebraic connectivity from the remaining largest connected component.

The first observation from Figure 6 is that there seems to be a slight increase in the value of the algebraic connectivity due to the random removal of nodes. On the contrary, from Figure 8 we observe that the value of the algebraic connectivity decreases due to the random removal of nodes.

To illustrate how different link densities influence the robustness, we show in Figures 7 and 9 the ratio of the number of nodes to the number of links after the scale-free graph of Barabási-Albert undergoes random node removals. We also see on Figures 8 and 9 that the gap between the algebraic connectivity before and after the removals reflects the decreasing degrees of the removed nodes. This illustrates the effect of the centrality of a node on the network robustness.

From the figures showing the ratio between the number of nodes and the number of links, we observe that for the
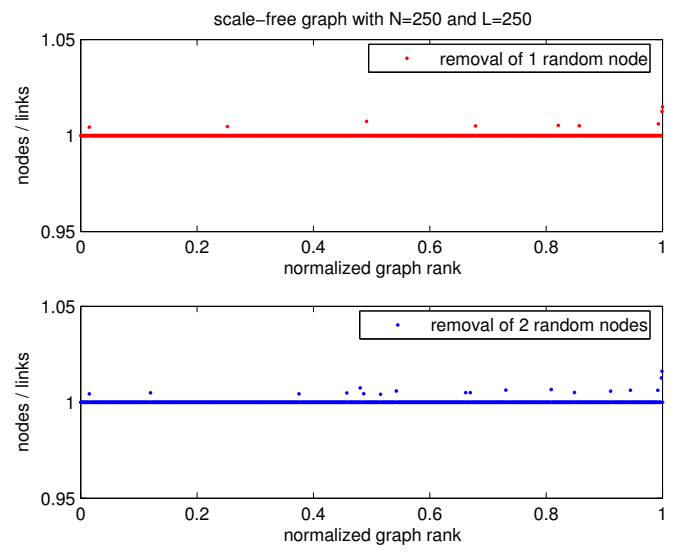

Fig. 7. The ratio of the number of nodes to the number of links after the scale-free graph of Barabási-Albert with 250 nodes and 250 links is exposed to the topological changes in the form of random node removal.

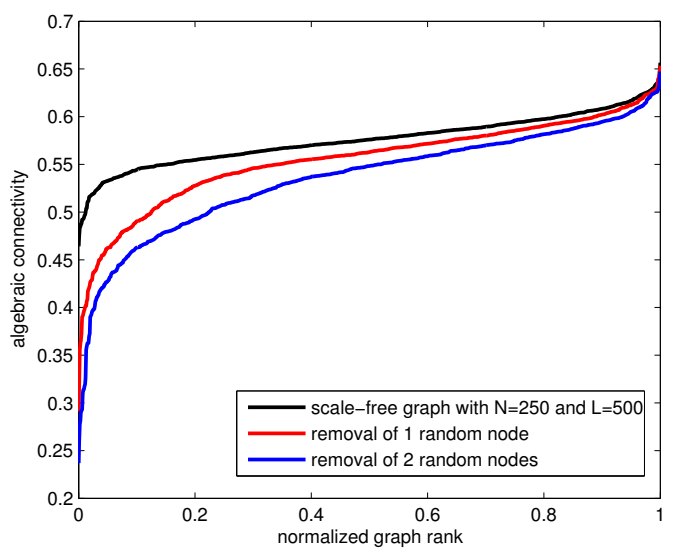

Fig. 8. Distribution of the algebraic connectivity for the scale-free graph of Barabási-Albert with 250 nodes and 500 links, with and without random nodes removal.

scale-free graph of Barabási-Albert with $N=L$, the random removal of nodes results in a similar topological structure, i.e. it results in a graph where the number of nodes approximately equals the number of links $N \approx L$. On the other hand, for the scale-free graph of Barabási-Albert with $N \ll L$, the random
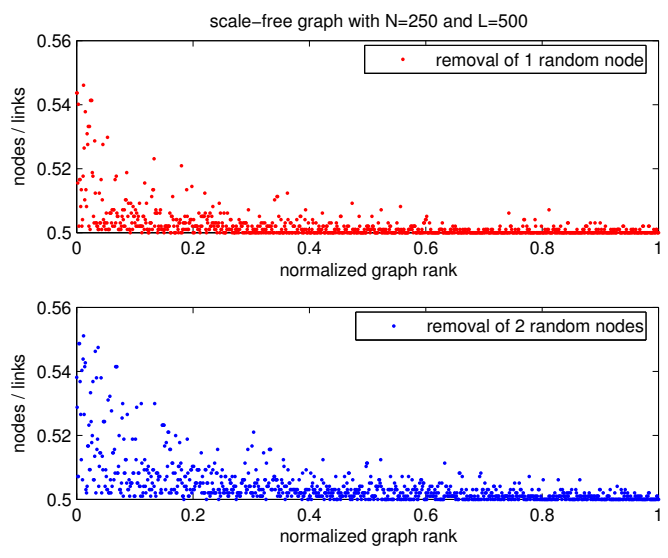

Fig. 9. The ratio of the number of nodes to the number of links after the scale-free graph of Barabási-Albert with 250 nodes and 500 links is exposed to the topological changes in the form of random node removal. 
removal of nodes results in a graph where the ratio of nodes to links increases compared to the original graph (where the ratio is 0.5 ). Note that for the scale-free graph of BarabásiAlbert with $N=250$ and $L=500$, the random removal of $i$ nodes results in a connected component with the number of nodes always equals to $N-i$.

The analysis of the random node failures allow us to conjecture the existence of networks belonging to two different robustness classes:

- The value of the algebraic connectivity slightly increases or remains the same: a graph has approximately the same number of nodes and links. After random node or link removal, a graph has on average the same number of nodes and links. This is either due to random nodes or random links whose removal results in a subgraph with the proportional number of nodes and links as in the original graph.

- The value of the algebraic connectivity decreases: a graph has more links than nodes. After random node or link removal, a graph has still on average more nodes than links. This decreasing algebraic connectivity is either due to

1) random nodes whose removal results in a subgraph with the node set that spans almost all nodes and consequently much less links than in the given graph.

2) random links whose removal results in a subgraph with the same node set as in the given graph.

Sparse and dense graphs hence will exhibit different behaviors of the algebraic connectivity under topological changes in the form of random node and link removal.

\section{CONClusion And Future Work}

Looking at the Erdôs-Rényi random graph, we observed that different values of the link probability $p$ and graph size $N$ may lead to identical probability distributions of the algebraic connectivity. Graph parameters of interest that correspond to an identical algebraic connectivity behavior, are deduced from the probabilities of a random graph being $k$-connected. This result supports a direct relationship between the algebraic connectivity and graph's robustness to node and link failures.

Furthermore, we have studied how the algebraic connectivity is affected by topological changes in the form of random node removal. For the Erdős-Rényi random graph, our findings confirm their well-know property: random removal of nodes results in a distribution similar to the distribution of the original random graph. Consequently, remaining largest connected components have similar structure and hence are equally robust to random removal of nodes. The random link removal strategy would most probably result in a decreasing distribution of the algebraic connectivity: remaining largest connected components most probably have unchanged set of nodes but a subset of the original links. Results obtained in the Erdős-Rényi random graph are confirmed but only by the small-world graph of Watts-Strogatz with higher link densities than its sparse counterparts (where a non-trivial robustness to random node failures is observed).
For the scale-free graph of Barabási-Albert, the distribution of the algebraic connectivity provides information on the type of failure the considered network has undergone: random node or link removal will increase the value of the algebraic connectivity only if the resulting subgraphs have approximately same number of nodes and links. On the other hand, random node or link removal will decrease the value of the algebraic connectivity only if the resulting subgraphs have a larger number of nodes than links.

Our results provide several immediate starting points for future work. First, we intend to use this insight for the problem of understanding how the algebraic connectivity is affected by other failure strategies. Second, we plan to apply the same reasoning to other network models. Third, it is extremely important to study the algebraic connectivity in real-world networks. Beside these three main goals, many invariants representing the robustness of networks are yet to be investigated thoroughly.

\section{REFERENCES}

[1] Albert, R., BARABAsi, A.-L., Statistical mechanics of complex networks, Reviews of Modern Physics 74, 47 (2002).

[2] BARABASI, A.-L. AND ALBERT, R., Emergence of scaling in random networks, Science 286, pp. 509-512 (1999).

[3] BollobÁs, B. And Thomason, A.G., Random graphs of small order. In random graphs., Annals of Discr. Math., pp.47-97 (1985).

[4] Bollobás, B., Modern graph theory, Springer-Verlag New York, Inc (1998).

[5] Bollobás, B., Random graphs, Cambridge University Press (2001).

[6] CAhn, R., Wide Area Network Design: Concepts \& Tools for Optimization, Morgan Kaufmann (1997).

[7] ERDős, P. AND RĖNYI, A., On random graphs, Publicationes Mathematicae 6, pp. 290-297 (1959).

[8] FIEDLER, M., Algebraic connectivity of graphs, Czechoslovak Mathematical Journal 23, pp. 298-305 (1973).

[9] Grone, R., MERris, R., Sunder, V.S., The Laplacian spectrum of a graph, SIAM Journal on Matrix Analysis 11, pp. 218-238 (1990).

[10] GRONE, R., On the geometry and Laplacian of a graph, Linear Algebra and its Applications 150, 167-178 (1991).

[11] Janson, S., KNuth, D.E., LuczaK, T. And Pittel, B., The birth of the giant component, Random Structures \& Algorithms, vol. 4, 233-358 (1993).

[12] JAMAKOVIC, A., UHLIG, S. On the relationship between the algebraic connectivity and graph's robustness to node and link failures, Proceedings of the 3rd IEEE EURO-NGI Conference on Next Generation Internet Networks, Norway (2007).

[13] Menger, K., Zur allgemeinen Kurventhoerie, Fundamenta Mathematicae 10:96-115, (1927).

[14] MERRIS, R., Laplacian matrices of graphs: a survey, Linear Algebra and its Applications 197 (1), pp. 143-176 (1994).

[15] Mohar, B., Alavi, Y., Chartrand, G., Oellermann, O.R. And SCHWEnK, A.J., The Laplacian spectrum of graphs, Graph Theory, Combinatorics and Applications 2, pp. 871-898 (1991).

[16] Mohar, B., Hahn, G., SABIDUssi, G., Some applications of Laplace eigenvalues of graphs, Graph Symmetry: Algebraic Methods and Applications, NATO ASI Ser. C 497, pp. 225-275 (1997).

[17] Newman, M.E.J., The structure and function of complex networks, SIAM Review 45, pp.167-256 (2002).

[18] Van Mieghem, P., Performance Analysis of Computer Systems and Networks, Cambridge University Press (2006).

[19] Watts, D.J. AND Strogatz, S.H., Collective dynamics of smallworld networks, Nature 393, pp. 440-442 (1999). 